\title{
Remoción de sulfuro empleando ozono como agente oxidante en aguas residuales de curtiembres
}

\author{
María Fernanda Umbarila-Ortega ${ }^{1}$ \\ Juan Sebastián Prado-Rodríguez ${ }^{2}$ \\ Rafael Nikolay Agudelo-Valencia ${ }^{3}$
}

Fecha de recepción: 8 de enero de 2019

Fecha de aprobación: 20 de marzo de 2019

\section{Resumen}

En este estudio se evaluó la remoción de sulfuros en aguas residuales del proceso de pelambre o depilado de pieles, utilizando ozono como agente oxidante. Se determinó el efecto del pH inicial sobre el proceso de remoción, para este fin se usó un diseño experimental completamente al azar, en el cual el pH del agua se varió entre 10,11, 12 y 13, el ozono fue dosificado a una tasa de $0,5 \mathrm{~g} \mathrm{~h}^{-1}$ por un periodo de 3 horas tomando muestras cada 30 minutos. Los ensayos fueron realizados para el agua residual real, suministrada por una curtiembre y agua residual sintética, a fin de comparar la remoción alcanzada en cada caso. El análisis de los resultados obtenidos permitió señalar que la mayor remoción se logró a $\mathrm{pH} 11$ en el agua residual real, alcanzando un valor de $77,78 \%$, aproximadamente $39 \%$ superior al alcanzado al mismo pH para el agua residual sintética. También se evaluó la cinética del proceso, obteniendo que los resultados se ajustan a una cinética de pseudo primer orden para la cual el coeficiente cinético fue de $-0,0001 \mathrm{~s}^{-1}$, por último, se observó que después de cada ensayo se presentó disminución $\mathrm{pH}$, lo cual confirma lo que químicamente debería suceder, formación de $\mathrm{H}_{2} \mathrm{SO}_{4}$ por la oxidación de sulfuros a sulfatos. Finalmente, se concluye que la remoción de sulfuros empleando ozono es una técnica aplicable para $\mathrm{pH}$ alcalino, permitiendo reducir tiempos de oxidación y convirtiéndose en una alternativa viable para aplicar en la industria del curtido de pieles.

Palabras clave: curtiembre; oxidación avanzada; ozono; pelambre; remoción; sulfuro.

\section{Sulfide removal using ozone as an oxidizing agent in tannery wastewater}

\section{Abstract}

In this study, the removal of sulfides in wastewater from the process of peeling or depilating skins was evaluated, using ozone as an oxidizing agent. The effect of the initial $\mathrm{pH}$ on the removal process was determined, for this purpose a completely randomized experimental design was used, in which the $\mathrm{pH}$ of the water was varied between 10, 11, 12 and 13, the ozone was dosed at a rate $0.5 \mathrm{~g} \mathrm{~h}^{-1}$ for a period of 3

\footnotetext{
1 Universidad Libre de Colombia (Bogotá-Distrito Capital, Colombia). mariaf-umbarilao@unilibre.edu.co. ORCID: 0000-0003-3776-8139.

2 Universidad Libre de Colombia (Bogotá-Distrito Capital, Colombia). juans-prador@unilibre.edu.co. ORCID: 0000-0001-6030-4311.

3 M. Sc. Universidad Libre de Colombia (Bogotá-Distrito Capital, Colombia). rafaeln.agudelov@unilibre.edu.co. ORCID: 0000-0002-6646-7725.
}

Revista Facultad de Ingeniería (Rev. Fac. Ing.) Vol. 28 (51), pp. 25-38. Abril-Junio 2019. Tunja-Boyacá, Colombia. L-ISSN: 0121-1129, e-ISSN: 2357-5328, DOI: 
hours taking samples every 30 minutes. The tests were performed for the real wastewater, supplied by a tannery and synthetic wastewater, in order to compare the removal achieved in each case. The analysis of the results obtained showed that the highest removal was achieved at $\mathrm{pH} 11$ in the actual wastewater, reaching a value of $77.78 \%$, approximately $39 \%$ higher than that reached at the same $\mathrm{pH}$ for the synthetic wastewater. The kinetics of the process were also evaluated, obtaining that the results are adjusted to a kinetic of pseudo first order for which the kinetic coefficient was $0,0001 \mathrm{~s}^{-1}$, finally, it was observed that after each test there was a decrease $\mathrm{pH}$, which confirms what chemically should happen, formation of $\mathrm{H}_{2} \mathrm{SO}_{4}$ by the oxidation of sulphides to sulfates. Finally, it is concluded that the removal of sulfides using ozone is a technique applicable for alkaline $\mathrm{pH}$, allowing to reduce oxidation times and becoming a viable alternative to apply in the leather tanning industry.

Keywords: advanced oxidation; clearing; ozone; removal; sulfur; tanning.

\section{Remoção de sulfeto empregando ozônio como agente oxidante em águas residuais de curtumes}

\section{Resumo}

Neste estudo avaliou-se a remoção de sulfetos em águas residuais do processo de pelagem ou depilado de peles, utilizando ozônio como agente oxidante. Determinou-se o efeito do $\mathrm{pH}$ inicial sobre o processo de remoção, para este fim usou-se um desenho experimental completamente ao azar, no qual o $\mathrm{pH}$ da água variou-se entre 10,11, 12 e 13, o ozônio foi dosificado a uma taxa de $0,5 \mathrm{~g} \mathrm{~h}^{-1}$ por um período de 3 horas tomando amostras a cada 30 minutos. Os ensaios foram realizados para a água residual real, subministrada por um curtume e água residual sintética, a fim de comparar a remoção alcançada em cada caso. A análise dos resultados obtidos permitiu assinalar que a maior remoção se logrou a pH 11 na água residual real, alcançando um valor de $77,78 \%$, aproximadamente $39 \%$ superior ao alcançado ao mesmo $\mathrm{pH}$ para a água residual sintética. Também avaliou-se a cinética do processo, obtendo com que os resultados se ajustem a uma cinética de pseudo primeira ordem para a qual o coeficiente cinético foi de $-0,0001 \mathrm{~s}^{-1}$, por último, observou-se que depois de cada ensaio apresentou-se diminuição do $\mathrm{pH}$, o qual confirma o que quimicamente deveria suceder, formação de $\mathrm{H}_{2} \mathrm{SO}_{4}$ pela oxidação de sulfetos a sulfatos. Finalmente, conclui-se que a remoção de sulfetos empregando ozônio é uma técnica aplicável para o pH alcalino, permitindo reduzir tempos de oxidação e convertendo-se em uma alternativa viável para aplicar-se na indústria do curtimento de peles.

Palavras chave: curtume; oxidação avançada; ozônio; pelagem; remoção; sulfeto.

\section{Para citar este artículo:}

M. F. Umbarila-Ortega, J. S. Prado-Rodríguez, and R. N. Agudelo-Valencia, "Remoción de sulfuro empleando ozono como agente oxidante en aguas residuales de curtiembres," Revista Facultad de Ingeniería, vol. 28 (51), pp. 25-38, Abr. 2019. DOI: https://doi.org/10.19053/01211129.v28.n51.2019.9081.

Esta obra está bajo licencia internacional Creative Commons Reconocimiento 4.0 


\section{INTRODUCCIÓN}

El sector productivo de curtido y teñido de cueros es reconocido por su considerable consumo de agua y altas cargas contaminantes, representadas en salinidad, materia orgánica, materia inorgánica, solidos disueltos y suspendidos, amoniaco, nitrógeno orgánico y algunos contaminantes específicos tales como sulfuros y cromo [1]. En los municipios de Villapinzón y Chocontá, Cundinamarca - Colombia, existen más de 100 empresas dedicadas al oficio del curtido de pieles [2]. Los vertimientos de las aguas residuales de este sector productivo son realizados al río Bogotá, dando lugar a problemas de contaminación de esta fuente hídrica superficial. Dentro del proceso de curtido y teñido de cueros, se realiza la operación de depilado, acción conocida en el sector curtidor como pelambre, esta etapa es responsable del $60 \%$ al 70 $\%$ de la carga contaminante del sector curtidor [3], cabe señalar que el agua residual expelida en el proceso de pelambre contiene alta concentración de sulfuro, Demanda Biológica de Oxígeno (DBO), Demanda Química de Oxígeno (DQO), Sólidos Suspendidos Totales (SST), y Sólidos Disueltos (SD) [1].

El principal problema de los sulfuros en el agua es su fácil reacción para formar sulfuro de hidrogeno, el cual es un compuesto que a exposiciones de menos de 30 minutos a concentraciones entre 1 y 11,6 ppmv causa sensibilidad bronquial en trabajadores sanos, daños en el sistema respiratorio y efectos en el sistema nervioso central de los animales [4]. Igualmente, según lo expuesto por Salas [5], el ion sulfuro, debido a su carácter reductor, disminuye el oxígeno presente en el agua y por lo tanto afecta drásticamente la vida acuática.

Reconocida la problemática que representa los sulfuros en el agua y la apremiante necesidad de aplicar tecnologías para su remoción, se han desarrollado e investigado diferentes alternativas para el tratamiento de aguas residuales contaminadas con sulfuro, siendo las más conocidas la oxidación catalítica con manganeso y aire [5], coagulación/floculación [6], oxidación electroquímica [7], electrocoagulación [8], oxidación con permanganato de potasio [9], las combinaciones entre luz UVC y oxígeno disuelto[10] y tratamiento anaerobio con ozono [11].

La remoción de sulfuros con ozono es una técnica prometedora debido a que es un elemento que no deja residuos secundarios en su reacción, eliminando un amplio tipo de componentes orgánicos presentes en el medio [12]. El ozono $\left(\mathrm{O}_{3}\right)$ es un compuesto con un potencial de oxidación de 2,07 en $\mathrm{V}$ (Voltios) a $25^{\circ} \mathrm{C}$, únicamente superado por el potencial de oxidación del flúor $(F) 3,03$, del radical hidroxilo $\left(\mathrm{HO}^{*}\right)$ 2,80 y del oxígeno atómico $(\mathrm{O})$ 2,42 [13]. Ramírez et al. (2016) en su estudio sobre el tratamiento de aguas residuales de curtiembre empleando ozono gaseoso, logró remociones de DQO, fenoles totales y color entre el 50\% - 95\% con un suministro inicial de ozono de $45 \mathrm{mg} / \mathrm{L}$ [14]. Investigaciones adicionales, como la de Sivagami et al. (2018) señalan que dosificando ozono a razón de $0,5 \mathrm{mg} \mathrm{O} / \mathrm{g}$ de DQO en $500 \mathrm{ml}$ de agua residual de curtiembre y 15 minutos de reacción, se alcanza una remoción entre el 10 $20 \%$ de la DQO presente en el efluente del tanque de sedimentación primario y entre el $40-50 \%$ de la DQO presente en el efluente del tanque de sedimentación secundario, así mismo, determinaron que la mayor eficacia del 
ozono se alcanzaba a pH 11 y que el tratamiento combinado de coagulación + aireación + ozono, alcanzaba una reducción de DQO entre 80 - 90 \% [15]. En el presente trabajo se empleó la oxidación de sulfuros con ozono, sin catalizador para la remoción de contaminantes para el tratamiento de aguas residuales de pelambren provenientes de una industria curtidora.

\section{Metodología}

\section{A. Área de estudio}

Las empresas curtidoras están situadas a las afueras del municipio de Villapinzón, Cundinamarca. Ubicadas en promedio a los $05^{\circ} 11^{\prime} 34.04^{\prime \prime} \mathrm{N}$ y $73^{\circ}$ 36 ' 57.49" O (151222,454 166108,511 metros en coordenadas planas) al sur occidente del municipio de Villapinzón, cuentan con una altitud media de 2671 m.s.n.m., y se encuentran justo al costado de la vía nacional número 55 y el Río Bogotá. A continuación, se presenta la ubicación de la industria del curtido en Villapinzón, y la ubicación de dicho municipio a nivel departamental y nacional. El mapa se realizó en el software ArcGis 10.3, se usaron mapas base integrados en el software y, Shapefiles de la Infraestructura de Datos Espaciales para el Distrito Capital (IDECA) y ArcGis Online, se utilizó la escala 1:55.000 y el sistema de coordenadas planas Magna Ciudad Bogotá. 


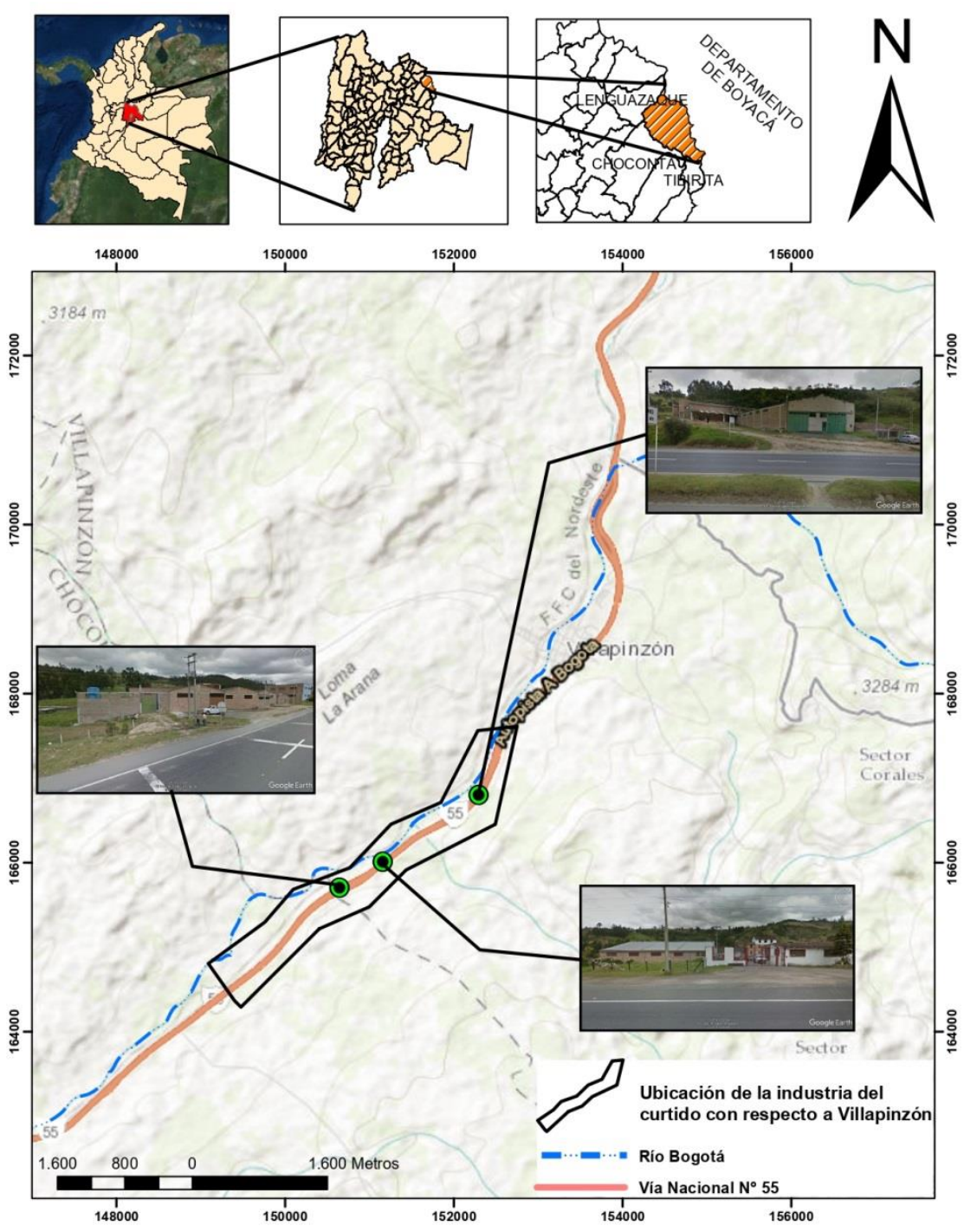

Fig 1. Ubicación de Villapinzón y la industria del curtido.

Por otro lado, las empresas ubicadas en el área de estudio, pertenecientes al sector industrial del curtido de pieles, en general realizan el mismo proceso para la obtención del cuero; dicho proceso se divide en cuatro etapas:

1) Ribera: Recepción de las pieles conservadas con cloruro de sodio y eliminación de componentes inservibles para la transformación en cuero. En la Ribera las pieles que llegan presentan la característica de ser de color verdoso debido a la sal o de presentar aún residuos de sangre. Para iniciar el proceso se limpian las pieles extrayendo de ellas el pelo, las proteínas y las grasas que se encuentran en la endodermis. Así mismo, dentro de esta etapa es llevado a cabo el proceso de pelambre o depilado, allí, se realiza un "ataque químico para eliminar el pelo y la epidermis, aumentar la separación entre las fibras de colágeno de la piel, destruir proteínas no estructurales, así como nervios, vasos sanguíneos, etc." [16].

2) Curtido: En este proceso se busca obtener una estabilidad química en la materia prima para evitar su descomposición sin importar que presente cambios de temperatura o humedad. Para llevar a cabo este proceso se 
hace uso de materiales de origen vegetal y sales de cromo. Una vez es finalizado este proceso a la piel se le denomina como cuero azul.

3) Recurtido: Teñido y Engrase: Las pieles adquieren cualidades físicas pertinentes para la comercialización de las mismas, como lo es el color.

4) Acabado: El cuero terminado es procesado para que reciba grabados 0 colores y así cumplir con los requerimientos del comprador, Para ello se implementan lacas o pigmentos.

\section{B. Materiales y reactivos}

El ajuste de $\mathrm{pH}$ fue realizado con $\mathrm{NaOH}(6 \mathrm{~N})$ y $\mathrm{HCl}(6 \mathrm{~N})$, el $\mathrm{pH}$ fue determinado haciendo uso de un potenciómetro Metrohm $781 \mathrm{pH} / \mathrm{lon}$ Meter. Para el análisis de sulfuros en las muestras fueron empleados los reactivos expuestos en los métodos estándar para análisis de agua potable y residual (método Standard Methods 4500-S²-F) [17], todos los reactivos fueron grado analítico, de la marca Merck Millipore.

\section{Diseño experimental}

El proceso experimental se realizó para determinar el efecto que ejerce el pH sobre la remoción de sulfuros en el tratamiento de aguas, se comparó la remoción del contaminante en aguas de pelambre obtenida en una curtiembre ubicada en el municipio de Villapinzón, Cundinamarca, contra la remoción en un agua residual sintética que solamente contenía sulfuro de sodio y cal.

Se evaluó la efectividad de la ozonización a distintos pH para cada una de las aguas, manteniendo constante el caudal de aire $\left(2,6 \mathrm{~L} \mathrm{~min}{ }^{-1}\right)$, dosis de ozono $\left(0.5 \mathrm{~g} \mathrm{~h}^{-1}\right)$ y tiempo de reacción (3 horas) de todos los ensayos. La recolección de muestras se hizo cada 30 minutos. El pH inicial de las aguas residuales utilizadas para los ensayos fue variado entre 10,11, 12 y 13, cada ensayo fue realizado por triplicado, es decir, en total se realizaron 24 experimentos y se recolectaron 7 muestras en cada uno de ellos. Así mismo, se determinó la tendencia cinética de la remoción de sulfuros con ozono para cada solución ideal y real en las distintas condiciones de $\mathrm{pH}$. La figura 2, corresponde al esquema metodológico empleado. 


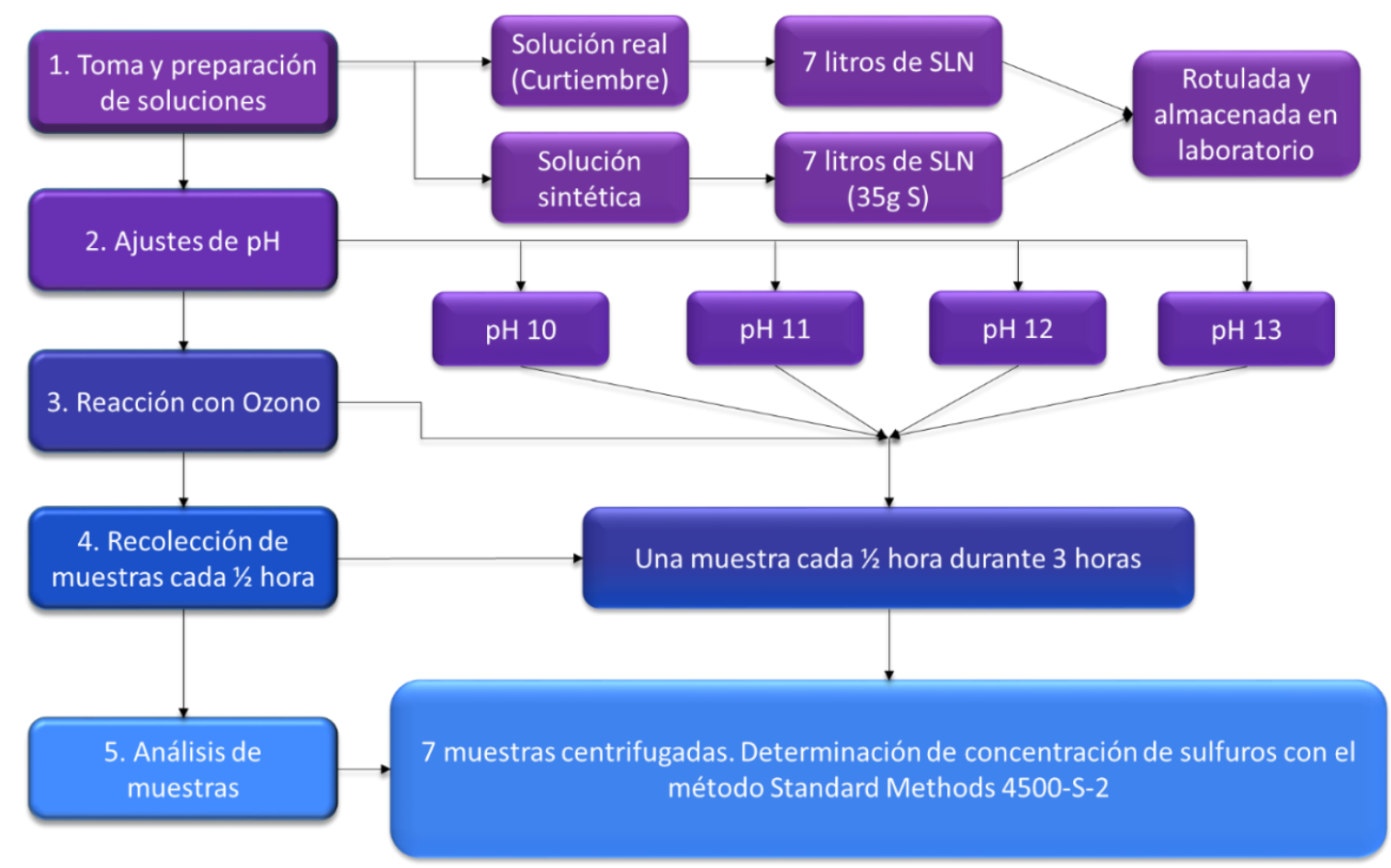

Fig. 2. Diseño metodológico.

\section{Equipo de ozono}

Para el suministro de ozono se empleó un equipo generador de ozono por efecto corona, el cual permito la dosificación de $0,5 \mathrm{~g} \mathrm{~h}^{-1}$ de ozono, este generador fue alimentado por aire a través de un compresor que entrego un flujo de 2,6 $\mathrm{L} \mathrm{min}^{-1}$ de aire. De igual forma, el difusor de piedra porosa generó burbujas de ozono con diámetros entre 2 y $4 \mathrm{~mm}$. A continuación, en la figura 3 , presenta las distintas partes del sistema de generación y suministro de ozono para los ensayos realizados.

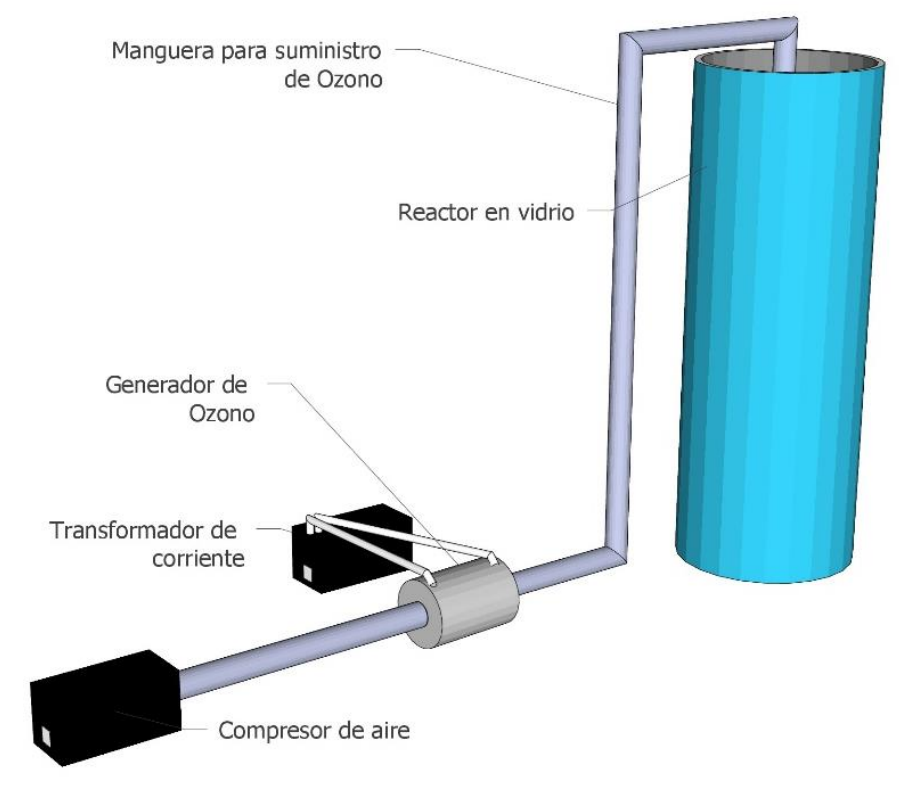

Fig. 3. Sistema empleado para la oxidación de sulfuros. 


\section{RESULTADOS Y ANÁLISIS}

En la Tabla 1 se observa como el pH final del agua vario en todos y cada uno de los ensayos, siendo mayor la diferencia de $\mathrm{pH}$ para los ensayos en los cuales se presentó la mayor remoción de sulfuro, es decir, para las muestras reales a pH 11 y 12, lo que confirma que la oxidación de sulfuro procede vía formación de ácido sulfúrico[18], como el único producto final detectable, y que, de acuerdo a la estequiometria de la reacción (1), 2,3 moles de ozono son requeridas para la formación de una mol de ácido sulfúrico.

$$
\mathrm{HS}^{-}+\mathrm{O}_{3} \rightarrow\left[\mathrm{HSOOO}^{-}\right]_{\mathrm{TS}} \rightarrow \mathrm{HSO}^{-}+\mathrm{O}_{2}
$$

Tabla 1. Comportamiento $\mathrm{pH}$ inicial y final.

\begin{tabular}{|c|c|c|c|c|}
\hline & \multicolumn{2}{|c|}{ Real } & \multicolumn{2}{c|}{ Sintética } \\
\hline $\boldsymbol{p H}$ & Inicial & Final & Inicial & Final \\
\hline 10 & 10,02 & 8,5 & 10,08 & 9,4 \\
\hline 11 & 11,02 & 8,72 & 11,03 & 9,6 \\
\hline 12 & 12,01 & 9,25 & 12 & 9,67 \\
\hline 13 & 13,01 & 12,95 & 13 & 12,87 \\
\hline
\end{tabular}

La Figura 4 presenta los porcentajes de remoción del sulfuro obtenidos para los distintos valores de $\mathrm{pH}$ inicial del agua, tanto para el agua residual sintética preparada en el laboratorio, como para el agua real tomada de la curtiembre. En el caso del agua residual sintética, se puede observar que el porcentaje de remoción de sulfuros disminuye a medida que se aumenta el $\mathrm{pH}$, lográndose una remoción del 48.7 \% para pH inicial de 10, en tres horas de reacción.

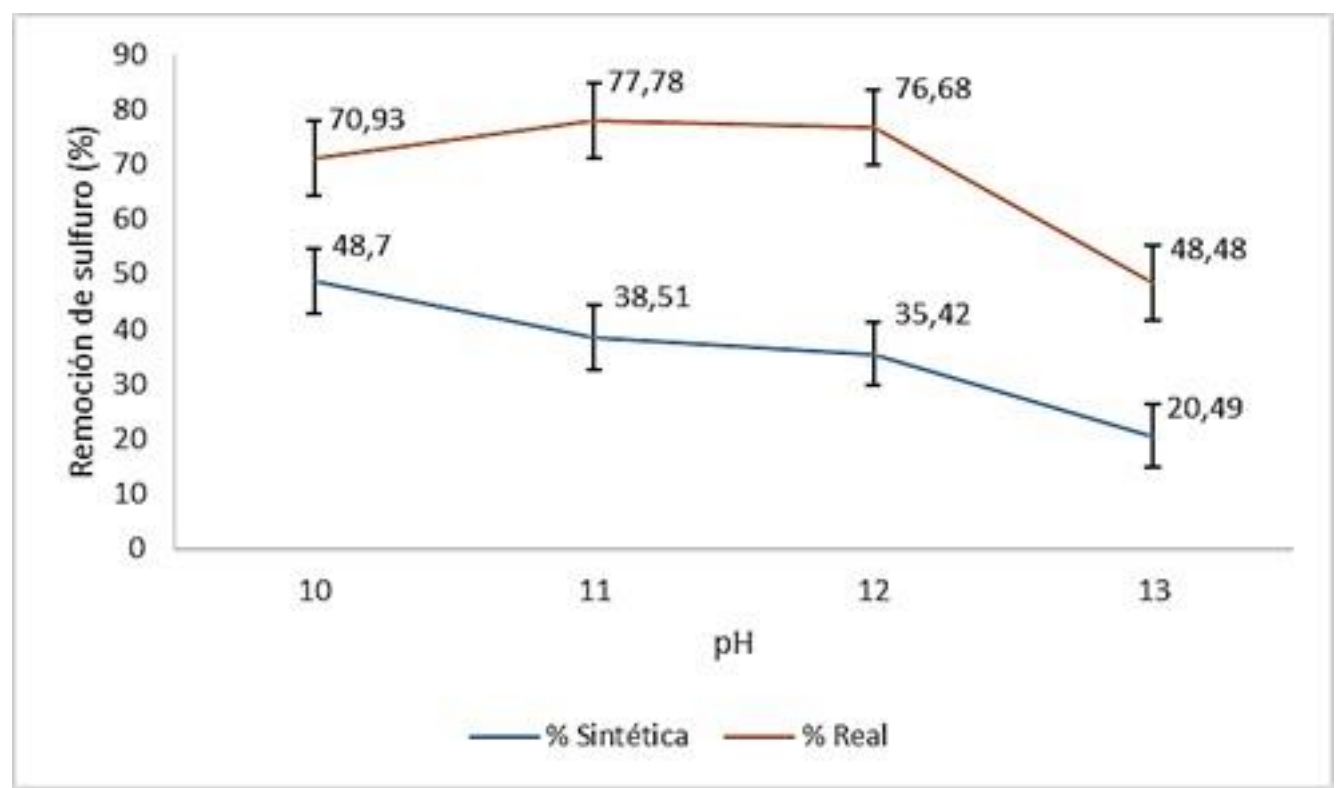

Fig. 4. Porcentaje de remoción de acuerdo al pH.

De la figura anterior también se observa que el porcentaje de remoción de sulfuros en todos los casos fue mayor para las aguas residuales reales (tomadas en la curtiembre) que para las aguas residuales sintéticas. Con respecto al agua residual real, el máximo porcentaje de remoción $(77.78 \%$ / $76.68 \%$ ) fue alcanzado para $\mathrm{pH}$ inicial entre 11 y 12 respectivamente, debido a 
la poca diferencia de los resultados, no se puede concluir cuál de estos dos valores de $\mathrm{pH}$ inicial del agua permitió alcanzar la mayor remoción pues no existe una diferencia estadísticamente significativa en la remoción promedio. Finalmente se determina que el $\mathrm{pH}$ presenta un efecto significativo sobre el proceso de oxidación de sulfuros con ozono, aumentando la remoción para pH inicial alcalino [15], por otra parte se observó que para pH inicial menor de 11 se presenta disminución de la efectividad del agente oxidante.

Como causa del aumento del porcentaje de remoción en la muestra real puede considerarse la presencia de sólidos en el agua, los cuales intervienen en el flujo del aire dentro del líquido, para favorecer la transferencia de masa de ozono desde la fase gaseosa a la fase liquida de manera que se logre saturar el agua residual con ozono y, por ende, asegurar la presencia y disponibilidad del oxidante en el agua para maximizar la remoción de sulfuros.

Con base en los resultados de remoción de sulfuro se determinó que la cinética de reacción para la oxidación de sulfuros se ajustó al modelo cinético de pseudo primer orden, se consideraron los modelos de pseudo primer y pseudo segundo orden debido a que el suministro de ozono permite suponer que la concentración de este en el agua fue constante. La Tabla 2 presenta los resultados del ajuste estadístico de los datos para determinar el tipo de modelo cinético que los describe.

Tabla 2. Tenencia cinética de la reacción para pH inicial del agua de 11 y 12.

\begin{tabular}{|c|c|c|}
\hline \multirow{2}{*}{ pH } & $\begin{array}{c}\text { Modelo cinético de pseudo } \\
\text { primer orden }\end{array}$ & $\begin{array}{c}\text { Modelo cinético de pseudo } \\
\text { segundo orden }\end{array}$ \\
\hline \multirow{2}{*}{11} & $\mathrm{~K}_{1}:-0.0001 \mathrm{~s}^{-1}$ & $\mathrm{~K}_{1}: 2^{*} 10^{-7} \mathrm{~L} \mathrm{mg}^{-1} \mathrm{~s}^{-1}$ \\
\cline { 2 - 3 } & $\mathrm{R}^{2}: 0.994$ & $\mathrm{R}^{2}: 0.9224$ \\
\hline \multirow{2}{*}{12} & $\mathrm{~K}_{1}:-0.0001 \mathrm{~s}^{-1}$ & $\mathrm{~K}_{2}: 2^{*} 10^{-7} \mathrm{~L} \mathrm{mg} \mathrm{m}^{-1} \mathrm{~s}^{-1}$ \\
\cline { 2 - 3 } & $\mathrm{R}^{2}: 0.996$ & $\mathrm{R}^{2}: 0.9589$ \\
\hline
\end{tabular}

La ecuación del modelo cinético de pseudo primer orden está representada por $\frac{d S}{d t}=-K_{1} * S$, en la ecuación anterior $\mathrm{K}_{1}$ es el coeficiente cinético de pseudo primer orden en $\mathrm{s}^{-1}$ y $\mathrm{S}$ es la concentración de sulfuros en el agua residual expresada en $\mathrm{mg} \mathrm{L}^{-1}$. La tendencia cinética del proceso evaluado fue realizada para agua residual real a $\mathrm{pH} 11$ y 12 , teniendo en cuenta que para este valor de $\mathrm{pH}$ inicial del agua se alcanzó el mayor porcentaje de remoción de sulfuros. En la figura 5 se presenta el gráfico correspondiente a la linealización de la cinética de pseudo primer orden, para agua residual real a $\mathrm{pH}$ inicial de 11, en este se observa claramente que los datos tienen tendencia lineal, lo que confirma el ajuste al modelo cinético analizado. 


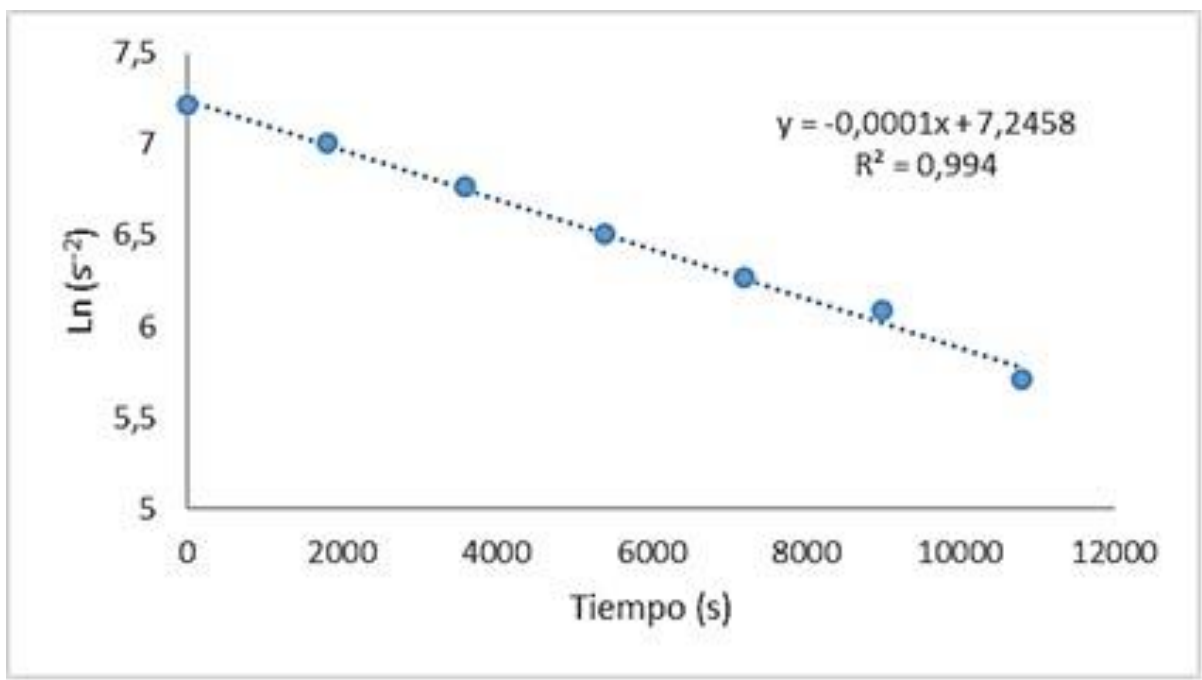

Fig. 5. Cinética de pseudo primer orden.

Las muestras tomadas según el diseño experimental fueron analizadas por medio de un ANOVA realzado en Excel Professional Plus 2016, a partir de éste se determinó la incidencia del $\mathrm{pH}$ sobre la remoción de sulfuros en el agua residual sintética y en el agua residual real, la hipótesis nula para el ANOVA fue que el porcentaje de remoción no varía con el $\mathrm{pH}$ para los ensayos realizados y, se estableció como un grado de confianza del 95\%, en la Tablas 3 y 4 se presenta el ANOVA para el análisis de los porcentajes de remoción promedio para los ensayos realizados.

Tabla 3. Análisis de Varianza ANOVA para el agua residual sintética.

\begin{tabular}{|l|c|r|c|r|r|r|}
\hline $\begin{array}{l}\text { Origen de las } \\
\text { variaciones }\end{array}$ & $\begin{array}{c}\text { Suma de } \\
\text { cuadrados }\end{array}$ & $\begin{array}{c}\text { Grados } \\
\text { de } \\
\text { libertad }\end{array}$ & $\begin{array}{c}\text { Promedio } \\
\text { de los } \\
\text { cuadrados }\end{array}$ & F & Probabilidad & $\begin{array}{c}\text { Valor crítico } \\
\text { para F }\end{array}$ \\
\hline Entre grupos & 1538,650881 & 3 & 512,8836269 & 4,714 & 0,035 & 4,066180551 \\
\hline $\begin{array}{l}\text { Dentro de los } \\
\text { grupos }\end{array}$ & 870,3577887 & 8 & 108,7947236 & & & \\
\hline Total & 2409,008669 & 11 & & & & \\
\hline
\end{tabular}

Tabla 4. Análisis de Varianza ANOVA para el agua residual real.

\begin{tabular}{|l|c|r|c|c|c|c|}
\hline $\begin{array}{c}\text { Origen de las } \\
\text { variaciones }\end{array}$ & $\begin{array}{c}\text { Suma de } \\
\text { cuadrados }\end{array}$ & $\begin{array}{c}\text { Grados } \\
\text { de } \\
\text { libertad }\end{array}$ & $\begin{array}{c}\text { Promedio de } \\
\text { los } \\
\text { cuadrados }\end{array}$ & F & Probabilidad & $\begin{array}{c}\text { Valor crítico } \\
\text { para F }\end{array}$ \\
\hline Entre grupos & 2112,011558 & 3 & 704,0038528 & 15,36 & 0,001106195 & 4,066180551 \\
\hline $\begin{array}{l}\text { Dentro de los } \\
\text { grupos }\end{array}$ & 366,6455333 & 8 & 45,83069167 & & & \\
\hline Total & 2478,657092 & 11 & & & & \\
\hline
\end{tabular}

EI ANOVA permite concluir que el valor del $\mathrm{pH}$ inicial del agua residual afecta la remoción de sulfuros cuando se hace uso de ozono como agente oxidante, lo que se verifica estadísticamente con los valores de probabilidad obtenidos de 0.035 y 0.011 los cual son menores que el valor alfa o grado de certeza seleccionado de 0.05 , dando validez a la hipótesis alterna, es decir, que las medias de los resultados si varían con respecto al $\mathrm{pH}$. 
Los valores promedio de porcentaje de remoción fueron analizados para determinar si existen diferencias estadísticamente significativas, entre los resultados para el agua residual sintética y el agua residual real, con el objeto de lograr esto se hizo uso de la prueba de comparación de valores medios por la distribución t-student. La Tabla 5 presenta los resultados de la prueba tstudent para la comparación de los valores medios de remoción de sulfuros.

Tabla 5. Análisis t-student del porcentaje de remoción.

\begin{tabular}{|l|r|r|}
\hline & Variable 1 & Variable 2 \\
\hline Media & 0,3578 & 0,68466387 \\
\hline Varianza & 0,01360977 & 0,01864704 \\
\hline Observaciones & 4 & 4 \\
\hline $\begin{array}{l}\text { Coeficiente de correlación de } \\
\text { Pearson }\end{array}$ & 0,75280536 & \\
\hline $\begin{array}{l}\text { Diferencia hipotética de las } \\
\text { medias }\end{array}$ & 0 & \\
\hline Grados de libertad & 3 & \\
\hline Estadístico t & $-7,18789014$ & \\
\hline $\mathrm{P}(T<=\mathrm{t})$ una cola & 0,00277443 & \\
\hline Valor crítico de t (una cola) & 2,35336343 & \\
\hline $\mathrm{P}(T<=\mathrm{t})$ dos colas & 0,00554886 & \\
\hline Valor crítico de t (dos colas) & 3,18244631 & \\
\hline
\end{tabular}

Como punto de partida para la prueba se planteó como hipótesis que el porcentaje de remoción era igual para todos los valores de $\mathrm{pH}$ y para los dos tipos de aguas residuales analizadas. El valor obtenido de $\mathrm{P}(\mathrm{T}<=\mathrm{t})$ para la prueba de dos colas, es menor al alfa establecido de 0.05 , indicando que la hipótesis planteada no fue válida, hecho que confirma que los valores obtenidos del porcentaje de remoción son diferentes para cada valor de $\mathrm{pH}$ y cada tipo de agua.

\section{CONCLUSIONES}

De acuerdo a los resultados se puede concluir que la mayor remoción de sulfuros en el agua se presentó para el agua residual real a pH 11 y alcanzó un valor de $77,78 \%$, además se encontró que el pH inicial óptimo para la remoción de sulfuros con ozono se encuentra entre 11 y 12, pero no se puede establecer con claridad cuál es exactamente el pH óptimo, debido a la baja diferencia estadística de los resultados. Se determinó que la tendencia cinética del proceso evaluado a valores de $\mathrm{pH}$ entre 11 y 12 se ajusta al modelo cinético de pseudo primer orden, para el cual el coeficiente cinético $(\mathrm{K})$ alcanzó un valor de $-0.0001 \mathrm{~s}^{-1}$. Se puede señalar que la reducción de $\mathrm{pH}$ presentada en cada uno de los ensayos se debe a la formación de ácido sulfúrico $\left(\mathrm{H}_{2} \mathrm{SO}_{4}\right)$ como único posible producto de oxidación con ozono. Finalmente, se considera que el aumento en el porcentaje de remoción de sulfuros observado para el agua residual real puede ser por efecto de la presencia de partículas en el agua, que favorecen la dispersión de las burbujas de ozono y por lo tanto maximizan la transferencia de masa aire/agua. 


\section{AGRADECIMIENTOS}

A la Universidad Libre de Colombia, a cada persona que aportó indirectamente en el proyecto como los instructores de laboratorio, a nuestros padres, amigos y conocidos que siempre nos brindaron apoyo incondicional y por supuesto a Dios.

Agradecimiento especial para la Sra. Jina Alexandra Ortega Pinzón, a la Sra. Fanny Stella Pinzón Delgado, a la Sra. Alba Victoria Rodríguez Suarez y al Sr. William Prado Guerra, por su gran apoyo y ánimo para la realización y culminación del proyecto.

Por último, agradecimientos a los profesores de las materias de investigación aplicada Diana Carolina Ospina Mora, Juan Antonio Aragón Moreno, Gina Paola González Angarita y Lida Esperanza Ruiz Martínez, quienes cada semestre nos guiaron en el desarrollo de nuestro proyecto y la construcción del presente artículo.

\section{REFERENCIAS}

[1] S. Jian, T. Wenyi, and C. Wuyong, "Kinetics of enzymatic unhairing by protease in leather industry," Journal of Cleaner Production, vol. 19 (4), pp. 325-331, Mar. 2011. DOl: https://doi.org/10.1016/j.jclepro.2010.10.011.

[2] Gobernación de Cundinamarca, Diagnóstico económico, ambiental y social de los curtidores de Choconta y Villapinzon (Cundinamarca). Bogotá D.C.: Gobernación de Cundinamarca, 2014.

[3] L. H. Wei Xu, and J. Zhang, "Hair-saving Enzyme-assisted Unhairing: Effects of Sodium Hydrosulfide and Peroxide.," in 32nd Congress of the International Union of Leather Technologists and Chemist Societies, Istanbul, 2009.

[4] Linde Ecuador S.A., Hoja de seguridad del material (SDS) sulfuro de hidrógeno - Producto químico e identificación de la 2012 empresa, Available: http://www.lindegas.ec/en/images/HOJA\%20DE\%20SEGURIDAD\%20SULFURO\%20DE\%20HIDROGENO tcm33998247.pdf.

[5] G. Salas, "Eliminación de sulfuros por oxidación en el tratamiento del agua residual de una curtiembre," Rev. Per. Quím. Ing. Quím, vol. 8 (1), pp. 49-54, 2005.

[6] Q. Imran, M. A. Hanif, M. S. Riaz, S. Noureen, T. M. Ansari, and H. N. Bhatti, "Coagulation/Flocculation of Tannery Wastewater Using Immobilized Chemical Coagulants," J. Appl. Res. Technol., vol. 10 (2), pp. 79-86, 2012.

[7] S. Sundarapandiyan, R. Chandrasekar, B. Ramanaiah, S. Krishnan, and P. Saravanan, "Electrochemical oxidation and reuse of tannery saline wastewater," Journal of Hazardous Materials, vol. 180 (1-3), pp. 197-203, Aug. 2010. DOI: https://doi.org/10.1016/i.jhazmat.2010.04.013.

[8] L. Bilińska, K. Blus, M. Gmurek, and S. Ledakowicz, "Coupling of electrocoagulation and ozone treatment for textile wastewater reuse," Chemical Engineering Journal, vol. 358, pp. 992-1001, Feb. 2019. DOI: https://doi.org/10.1016/i.cej.2018.10.093.

[9] D. E. Brune, and R. L. Perez, "A continuously regenerated greensand filter for H 2 S removal from individual water supplies," J. Environ. Sci. Heal. Part A Environ. Sci. Eng. Toxicol., vol. 25 (1), pp. 120, Jan. 1990.

[10] Y. Tzvi, and Y. Paz, "Highly efficient method for oxidation of dissolved hydrogen sulfide in water, utilizing a combination of UVC light and dissolved oxygen," Journal of Photochemistry and Photobiology A Chemistry, vol. 372, pp. 63-70, Mar. 2019. DOI: https://doi.org/10.1016/i.jphotochem.2018.12.005.

[11] S. Venkatesh, K. Venkatesh, and A. R. Quaff, "Dye decomposition by combined ozonation and anaerobic treatment: Cost effective technology," Journal of Applied Research and Technology, vol. 15 (4), pp. 340-345, Aug. 2017. DOI: https://doi.org/10.1016/j.jart.2017.02.006.

[12] F. J. Rodríguez Vidal, Procesos de potabilización del agua e influencia del tratamiento de ozonización. Madrid: Ediciones Díaz de Santos, 2003.

[13] J. Pey Clemente, "Aplicación de procesos de oxidación avanzada (fotocatálisis solar) para tratamiento y reutilización de efluentes textiles," Thesis, Universidad Politécnica de Valencia, 2008.

[14] N. Ramírez-Ramírez, M. C. Espinosa-Lloréns, L. A. Fernández-García, E. Véliz-Lorenzo, and Y. Ramos-Rodríguez, "Treatment with Ozone of Wastewater Containing Tannins from Vegetal Tannery," Water Technol. Sci. (in Spanish), vol. 7 (3), pp. 53-73, 2016.

[15] K. Sivagami, K. P. Sakthivel, and I. M. Nambi, "Advanced oxidation processes for the treatment of tannery wastewater," Journal of Environmental Chemical Engineering, vol. 6 (3), pp. 3656-3663, Jun.

Revista Facultad de Ingeniería (Rev. Fac. Ing.) Vol. 28 (51), pp. 25-38. Abril-Junio 2019. Tunja-Boyacá, Colombia. L-ISSN: 0121-1129, e-ISSN: 2357-5328, DOI: 
2018. DOI: https://doi.org/10.1016/j.jece.2017.06.004.

[16] Instituto Nacional de Ecología, Manual de procedimientos para el manejo adecuado de los residuos de curtiduría. Ciudad de México: Instituto Nacional de Ecología, 1999.

[17] A. P. H. Association, Standard Methods For the Examination of Water and Wastewater. Maryland, 2012.

[18] C. Von Sonntag, and U. Von Gunten, Chemistry of Ozone in Water and Wastewater Treatment. London: IWA Publishing, 2012. 